\title{
Implementation of IT Project Management Control and Achieving the Control Objectives using Hybrid Extended Methodology
}

\author{
Anirudh Arunan \\ Department of computer \\ Science and Engineering \\ Vellore Institute of Technology, \\ Vellore, 632014 \\ India
}

\author{
Krishan Kumar \\ Department of computer \\ Science and Engineering \\ Vellore Institute of Technology, \\ Vellore, 632014 \\ India
}

\author{
Manjula $\mathrm{R}$. \\ Department of computer \\ Science and Engineering \\ Vellore Institute of Technology, \\ Vellore, 632014 \\ India
}

\begin{abstract}
This paper looks into the various aspects and components of project management control with a view to identify a new methodology using a hybrid model to achieve control objectives. Classical approaches to project management control are explored. The best known methods of Project management are Water fall ${ }^{3}$ and Scrum methods ${ }^{3}$. However in a real world scenario, neither of these methodologies may be the best fit. Hence with the help of empirical project data an attempt is made to analyze options and see how effective is a hybrid model in achieving optimal control.

To achieve this, additional control criteria like quality control and development of reusable components etc are used. The analysis is carried out using specific IT project implementation data of a medium size project. The project was implemented using standard Software Development Life Cycle (SDLC) techniques.
\end{abstract}

\section{General Terms}

IT project management, software development

\section{Keywords}

IT project management, Control objectives, Hybrid extended methodology, software development life cycle

\section{INTRODUCTION}

In traditional project management, monitoring project progress against schedules and budgets is the focus and priority of managers. In the modern approach, many additional variables of control at different stages of the project progress (e.g. user contributions, efficiency of team task completion, performance of the team, quality of delivery etc.) are also considered.

One of the major challenges faced by project owners is to get assurances that business objectives are being met without compromising on compliance of standards and quality. This is achieved by appointing specialist consults to develop and manage extensive control frameworks and use specialist auditors to ensure full compliance to the control frame work.

According to the Project Management Body of Knowledge (PMBOK) ${ }^{4}$, "the Monitoring and Control Process Group consists of those processes that are performed to observe project execution so that potential problems can be identified in a timely manner and corrective action can be taken, when necessary, to control the execution of the project."
Project Monitoring and Control activities take place in parallel with Project Execution Process Group activities. This will enable monitoring and controlling of the project by applying appropriate level of corrective action while the project work is being executed, to avoid potential delays and cost over-run.

The project progress is monitored and measured at predefined intervals against the mile stones in project plan. This is done to ensure that the project is executed within acceptable level of variances of scope, schedule and cost. Also, the identified risks are monitored continuously and corrective actions are taken as necessary.

The regular monitoring and controlling activities will enable finding of issues proactively ahead of time and help in taking corrective actions in line with the project control objectives. Some corrective actions will require revisiting Planning Process Group resulting in updating the Project Management Plan. These activities will enable mitigating any delays and helps in bringing back the project back in track in line with project objectives and time lines.

\section{PURPOSE}

Purpose of this research paper is to develop a hybrid project control model to achieve control objectives of a project. Control processes include:

1. Controlling the Project Work

2. Integrated Change Control

3. Scope Control

4. Quality Control

5. Schedule Control

6. Resource control

7. Cost Control

\section{DISCUSSIONS}

\subsection{Current Models and Approaches}

Most project managers either adopt Agile or Waterfall approach to project management to achieve the project management and control objectives

The waterfall approach is based on phases that consist of "Plan, Do, Check and Act" method. On the other hand, Agile approach is based on a modular approach that require work to be planned as per the Acceptance Process and in concurrent form wherever possible. 
Waterfall $^{3}$ is a linear approach to software development. In this methodology, the sequence of events is something like:

1. Gather and document requirements

2. Design

3. Code and unit test

4. Perform system testing

5. Perform user acceptance testing (UAT)

6. Fix any issues

7. Deliver the finished product

Agile ${ }^{3}$ is an iterative, team-based approach to development. This approach emphasizes the rapid delivery of an application in complete functional components. Rather than creating tasks and schedules, all time is "time-boxed" into phases called "sprints." Each sprint has a defined duration (usually in weeks) with a running list of deliverables, planned at the start of the sprint. Deliverables are prioritized by business value as determined by the customer. If all planned work for the sprint cannot be completed, work is reprioritized and the information is used for future sprint planning.

The typical stages of a project as per $\mathrm{PMBOK}^{3}$ are "Initiate, Plan, Execute, Control \&Complete".

COBIT (Control Objectives for Information and Related Technologies) framework ${ }^{1}$ is used to provide a set of controls for implementing IT projects. Edward W. N. Bernroider ${ }^{1}$ discusses how to achieve control objectives in an IT project using COBIT.

\section{OBJECTIVE}

This paper is an attempt to see how a Hybrid model (part use of Agile and part use of Scrum) approach to better achieve Project management and control objectives

Detailed analysis using empirical data to be carried out to establish the concept and validity of it

\subsection{Key Control objectives}

Below are the list of key project management areas and their performance control objectives ${ }^{1}$.

\begin{tabular}{|c|c|}
\hline $\begin{array}{r}\text { Sl } \\
\text { No }\end{array}$ & $\begin{array}{l}\text { Project } \\
\text { Management } \\
\text { Areas } \\
\end{array}$ \\
\hline 1 & $\begin{array}{ll}\text { Business } & \& \\
\text { Regulatory } & \\
\text { Environment } & \end{array}$ \\
\hline
\end{tabular}

\section{Performance Control} Objectives

Business revenue/benefit will be realized

\begin{tabular}{|l|l|}
2 & $\begin{array}{l}\text { Scope \& Change } \\
\text { Control }\end{array}$ \\
\hline 3 & $\begin{array}{l}\text { Time (Schedule) } \\
\text { Management }\end{array}$ \\
\hline 4 & $\begin{array}{l}\text { Quality } \\
\text { Inspection \& }\end{array}$ \\
\hline 5 & Cost Management \\
\hline 6 & $\begin{array}{l}\text { Communication \& } \\
\text { Reporting }\end{array}$ \\
\hline 7 & $\begin{array}{l}\text { People } \\
\text { Management }\end{array}$ \\
\hline 8 & $\begin{array}{l}\text { Procurement and } \\
\text { Contracts }\end{array}$ \\
\hline 9 & $\begin{array}{l}\text { Risk and Issue } \\
\text { Management }\end{array}$ \\
\hline
\end{tabular}

Impact of any changes are understood and managed

Key upcoming milestones will be met

Technical, standardization and quality requirements are met

Actual \& forecast vs planned cost variances are authorized

Stakeholders happy \& team / suppliers are performing at the desired level

Issue impacts are understood and improvements planned

\subsection{The Criteria to Assess Achievement of the above control Objectives:}

The basis for assessing whether control objectives are met is the maturity of control processes, in line with their criticality. Processes which are assessed/rated as 'Not achieved' will require time based and documented improvement plans. The normally used criteria and rating are

1. Nothing in place

2. Informal (not documented)

3. Working, but not fully documented or tested or regularly monitored

4. Documented and in place, but not tested or monitored

5. Documented and tested. Also monitored and reported

6. Fully Optimized and gone through improvement processes to improve value

\subsection{Control gap assessment}

The basis for the control gap assessment is a matrix of critical process controls. These processes span across the life cycle of the project with key gaps included in the project risk register. Some examples are listed below

\begin{tabular}{|l|l|l|l|l|l|}
\hline Planning stage & \multicolumn{1}{|c|}{ Design stage } & Implementation/development stage & Testing stage & $\begin{array}{l}\text { Roll Out } \\
\text { stage }\end{array}$ & $\begin{array}{c}\text { Maintenance } \\
\text { /Support stage }\end{array}$ \\
\hline $\begin{array}{l}\text { Acceptance of the } \\
\text { Project and } \\
\text { Funding }\end{array}$ & $\begin{array}{l}\text { Assessment of } \\
\text { Business } \\
\text { requirements }\end{array}$ & Development of success criteria & $\begin{array}{l}\text { Development of continuous } \\
\text { Improvement and plan }\end{array}$ \\
\hline $\begin{array}{c}\text { Definition of } \\
\text { Projects phases } \\
\text { and components }\end{array}$ & $\begin{array}{l}\text { Design and } \\
\text { develop Project } \\
\text { Components }\end{array}$ & Agree Change Control Process and framework & $\begin{array}{l}\text { Define User } \\
\text { Acceptance } \\
\text { Process and } \\
\text { criteria }\end{array}$ & $\begin{array}{l}\text { Define } \\
\text { Operations } \\
\text { Acceptance } \\
\text { Process }\end{array}$ \\
\hline $\begin{array}{l}\text { Project Schedule } \\
\text { Requirements }\end{array}$ & $\begin{array}{l}\text { Baseline Project } \\
\text { Schedule }\end{array}$ & Detailed Schedule Management & $\begin{array}{l}\text { Schedule the } \\
\text { Completion } \\
\text { Check List }\end{array}$ & $\begin{array}{l}\text { Ongoing } \\
\text { Maintenance } \\
\text { Schedule }\end{array}$ \\
\hline
\end{tabular}




\begin{tabular}{|c|c|c|c|c|c|}
\hline $\begin{array}{l}\text { Project Concept } \\
\text { and Performance } \\
\text { Requirements }\end{array}$ & $\begin{array}{l}\text { Review and } \\
\text { Approval of the } \\
\text { overall design }\end{array}$ & Quality Assurance (QA)Process & $\begin{array}{l}\text { Carry out } \\
\text { Inspections } \\
\text { \& Quality } \\
\text { Review }\end{array}$ & $\begin{array}{l}\text { Project } \\
\text { completion } \\
\text { Check List }\end{array}$ & $\begin{array}{l}\text { Quality } \\
\text { Assurance } \\
\text { Process }\end{array}$ \\
\hline Capital Budgeting & $\begin{array}{l}\text { Forecast Cost } \\
\text { and Schedule }\end{array}$ & \multicolumn{2}{|c|}{ Cost Control/cost optimization } & Final Payment & $\begin{array}{l}\text { Operation and } \\
\text { maintenance } \\
\text { Budget }\end{array}$ \\
\hline \multicolumn{3}{|c|}{ Project Management Plan and Resourcing } & Staff optimizing & $\begin{array}{l}\text { Planning } \\
\text { Operations Staff }\end{array}$ & $\begin{array}{c}\text { Ongoing } \\
\text { Requirements / } \\
\text { Skills Review }\end{array}$ \\
\hline $\begin{array}{l}\text { Defining Project } \\
\text { Reporting } \\
\text { Requirements }\end{array}$ & $\begin{array}{l}\text { Project Status } \\
\text { reporting }\end{array}$ & $\begin{array}{c}\text { Define variance - Project Cost, and } \\
\text { Schedule }\end{array}$ & $\begin{array}{l}\text { Project Quality } \\
\text { Performance }\end{array}$ & $\begin{array}{l}\text { Project Closure } \\
\text { Performance }\end{array}$ & $\begin{array}{l}\text { Financial } \\
\text { Reporting }\end{array}$ \\
\hline $\begin{array}{l}\text { Outsourcing/Exte } \\
\text { rnal Contracting } \\
\text { Options }\end{array}$ & $\begin{array}{l}\text { Partner } \\
\text { Qualification / } \\
\text { RFP Process }\end{array}$ & Vendor Selection & $\begin{array}{l}\text { Contract } \\
\text { Compliance } \\
\text { Review }\end{array}$ & $\begin{array}{l}\text { Trouble } \\
\text { shooting/debugg } \\
\text { ing }\end{array}$ & $\begin{array}{l}\text { Vendor } \\
\text { Qualification }\end{array}$ \\
\hline $\begin{array}{l}\text { Project Risk } \\
\text { Management } \\
\text { Planning }\end{array}$ & \multicolumn{3}{|c|}{ Risk and Issue Tracking and achieve Resolution } & $\begin{array}{l}\text { Confirm Issue } \\
\text { Resolution }\end{array}$ & $\begin{array}{l}\text { Continuous Issue } \\
\text { Management } \\
\text { Process }\end{array}$ \\
\hline $\begin{array}{l}\text { Acceptance of the } \\
\text { Project and } \\
\text { Funding }\end{array}$ & $\begin{array}{l}\text { Assessment of } \\
\text { Business } \\
\text { requirements }\end{array}$ & \multicolumn{2}{|l|}{ Development of success criteria } & \multicolumn{2}{|c|}{$\begin{array}{l}\text { Development of continuous } \\
\text { Improvement and plan }\end{array}$} \\
\hline $\begin{array}{c}\text { Definition of } \\
\text { Projects phases } \\
\text { and components }\end{array}$ & $\begin{array}{l}\text { Design and } \\
\text { develop Project } \\
\text { Components }\end{array}$ & \multicolumn{2}{|c|}{ Agree Change Control Process and framework } & $\begin{array}{l}\text { Define User } \\
\text { Acceptance } \\
\text { Process and } \\
\text { criteria }\end{array}$ & $\begin{array}{l}\text { Define } \\
\text { Operations } \\
\text { Acceptance } \\
\text { Process }\end{array}$ \\
\hline $\begin{array}{l}\text { Project Schedule } \\
\text { Requirements }\end{array}$ & $\begin{array}{l}\text { Baseline Project } \\
\text { Schedule }\end{array}$ & \multicolumn{2}{|l|}{ Detailed Schedule Management } & $\begin{array}{l}\text { Schedule the } \\
\text { Completion } \\
\text { Check List }\end{array}$ & $\begin{array}{l}\text { Ongoing } \\
\text { Maintenance } \\
\text { Schedule }\end{array}$ \\
\hline $\begin{array}{c}\text { Project Concept } \\
\text { and Performance } \\
\text { Requirements }\end{array}$ & $\begin{array}{l}\text { Review and } \\
\text { Approval of the } \\
\text { overall design }\end{array}$ & Quality Assurance (QA)Process & $\begin{array}{l}\text { Carry out } \\
\text { Inspections } \\
\text { \& Quality } \\
\text { Review }\end{array}$ & $\begin{array}{l}\text { Project } \\
\text { completion } \\
\text { Check List }\end{array}$ & $\begin{array}{l}\text { Quality } \\
\text { Assurance } \\
\text { Process }\end{array}$ \\
\hline Capital Budgeting & $\begin{array}{l}\text { Forecast Cost } \\
\text { and Schedule }\end{array}$ & Cost Control/cost optim & ion & Final Payment & $\begin{array}{l}\text { Operation and } \\
\text { maintenance } \\
\text { Budget }\end{array}$ \\
\hline \multicolumn{3}{|c|}{ Project Management Plan and Resourcing } & Staff optimizing & $\begin{array}{l}\text { Planning } \\
\text { Operations Staff }\end{array}$ & $\begin{array}{l}\text { Ongoing } \\
\text { Requirements / } \\
\text { Skills Review }\end{array}$ \\
\hline $\begin{array}{l}\text { Defining Project } \\
\text { Reporting } \\
\text { Requirements }\end{array}$ & $\begin{array}{l}\text { Project Status } \\
\text { reporting }\end{array}$ & $\begin{array}{l}\text { Define variance - Project Cost, and } \\
\text { Schedule }\end{array}$ & $\begin{array}{l}\text { Project Quality } \\
\text { Performance }\end{array}$ & $\begin{array}{l}\text { Project Closure } \\
\text { Performance }\end{array}$ & $\begin{array}{l}\text { Financial } \\
\text { Reporting }\end{array}$ \\
\hline $\begin{array}{l}\text { Outsourcing/Exte } \\
\text { rnal Contracting } \\
\text { Options }\end{array}$ & $\begin{array}{l}\text { Partner } \\
\text { Qualification / } \\
\text { RFP Process }\end{array}$ & Vendor Selection & $\begin{array}{l}\text { Contract } \\
\text { Compliance } \\
\text { Review }\end{array}$ & $\begin{array}{l}\text { Trouble } \\
\text { shooting/debugg } \\
\text { ing }\end{array}$ & $\begin{array}{l}\text { Vendor } \\
\text { Qualification }\end{array}$ \\
\hline $\begin{array}{l}\text { Project Risk } \\
\text { Management } \\
\text { Planning }\end{array}$ & Risk and Issue $\mathrm{T}_{1}$ & king and achieve Resolution & & $\begin{array}{l}\text { Confirm Issue } \\
\text { Resolution }\end{array}$ & $\begin{array}{l}\text { Continuous Issue } \\
\text { Management } \\
\text { Process }\end{array}$ \\
\hline
\end{tabular}

\section{PROPOSED MODEL}

In the proposed model, upon completion of the planning and design stage, the next four stages (Design, implementation, testing, roll out and support) can be implemented in an agile manner running all these stages in parallel in a modular way.
Based on empirical data derived from the case study in Appendix 1 below clearly shows the advantage of the Hybrid model. 


\subsection{Water Fall Method - Roll out time period is 14 Months}

$\begin{array}{llllllllllllll}\text { M } & M & M & M & M & M & M & M & M & M & M & M & M & M\end{array}$

$\begin{array}{llllllllllllll}1 & 2 & 3 & 4 & 5 & 6 & 7 & 8 & 9 & 10 & 11 & 12 & 13 & 14\end{array}$

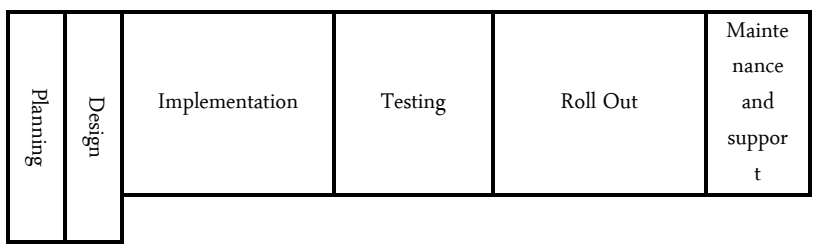

\subsection{Hybrid Extended Model - Roll out time period is 10 Months}

$\begin{array}{llllllllll}\text { M } & \text { M } & \text { M } & \text { M } & \text { M } & \text { M6 } & \text { M } & \text { M } & \text { M } & \\ 1 & 2 & 3 & 4 & 5 & \text { M } & 7 & 8 & 9 & 1\end{array}$

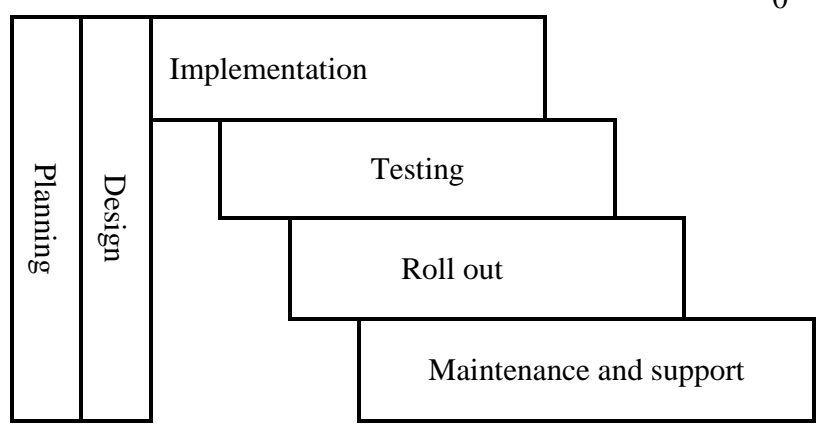

It is obvious that by adopting a Hybrid model, on an average $40 \%$ (10 months instead of 14 months) reduction in project timelines can be achieved by implementing proper control.

\section{CONCLUSIONS}

It is very obvious that in complex software implementation projects where system roll out is required across multiple locations, hybrid model is most appropriate for achieving control and delivery objectives. This is also applicable in the case of IT projects were reusable components are to be deployed.

However there will be a slightly higher effort in testing of the solution as the roll out starts before the completion of the full development cycle. However the overall saving in project time line and hence costs will more than make up for the additional testing efforts.

\section{REFERENCES}

[1] IT project management control and the Control Objectives for IT and related Technology (CobiT) framework by Edward W. N. Bernroider and Milen Ivanov, Article in International Journal of Project Management, April 2011

[2] Modelling project management performance. International Journal of Quality \& Reliability Management, 20, 229-254 by BRYDE, D. J. 2003

[3] Waterfall Vs Agile: Which is the Right development methodology for your Project? http://www.seguetech.com/waterfall-vs-agile-which-isthe-right-development-methodology-for-your-project/
[4] A Guide to the Project Management Body of Knowledge (PMBOK® Guide)_-Fifth Edition, PMI Institute

[5] Project management: cost, time and quality, two best guesses and a phenomenon, its time to accept other success criteria by Roger Atkinson Department of Information Systems, The Business School, Bournemouth University, Talbot Campus, Poole, Dorset BH12 5BB, UK

[6] Defining and measuring project success by Danie van der Westhuizen, Department Information Systems, Faculty of Business, University of Southern Queensland - Wide Bay Campus, Hervey Bay, Queensland, Australia

[7] Project Controls: What is it and why is it important? at http://www.projectcontrolsonline.com/Home/Defini tionandImportanceofProjectControls.aspx

[8] Breaking through the Project Fog by James Norrie published by John Wiley and Sons

[9] Portfolios of Control Modes and IS Project Management by Laurie S. Kirsch, Katz Graduate School of Business, The University of Pittsburgh, Pittsburgh, Pennsylvania

[10] Project Management Methodologies: A Comparative Analysis, by M. M. Chin (Kuala Lumpur Infrastructure University College), (PhD) and A. C. Spowage, (University of Nottingham Malaysia Campus), (PhD) and E. H. Yap, (University of Nottingham Malaysia Campus), (PhD)

[11] Project Management Methodologies: A Review of the Literature by Pamela McClinton

[12] Project Controls to Minimize Cost and Schedule Overruns: A Model, Research Agenda, and Initial Results David N. Ford, James M. Lyneis, and Timothy R.B. Taylor

[13] Statistical Project Control at http://www.projectmanagement.ugent.be/?q=research/pr oject_control/spc

[14] Impact of Real-time Project Control on Capital Project Cost and Schedule Performance by David Grau and Amin Abbaszadegan, School of Sustainable Engineering and the Built Environment; Arizona State University; Tempe AZ, USA david.grau@asu.edu

\section{APPENDIX}

Below is the project plan of an ERP implementation that is rolled out across 6 locations. As per the project plan developed in a Hybrid model, the first location takes double the roll out time of remaining locations. Also, as many tasks are carried out in parallel, overall project completion time is substantially reduced.

But the implementation will become complex requiring strong project management skills and additional project resources for parallel roll out. 
ERP implementation Plan

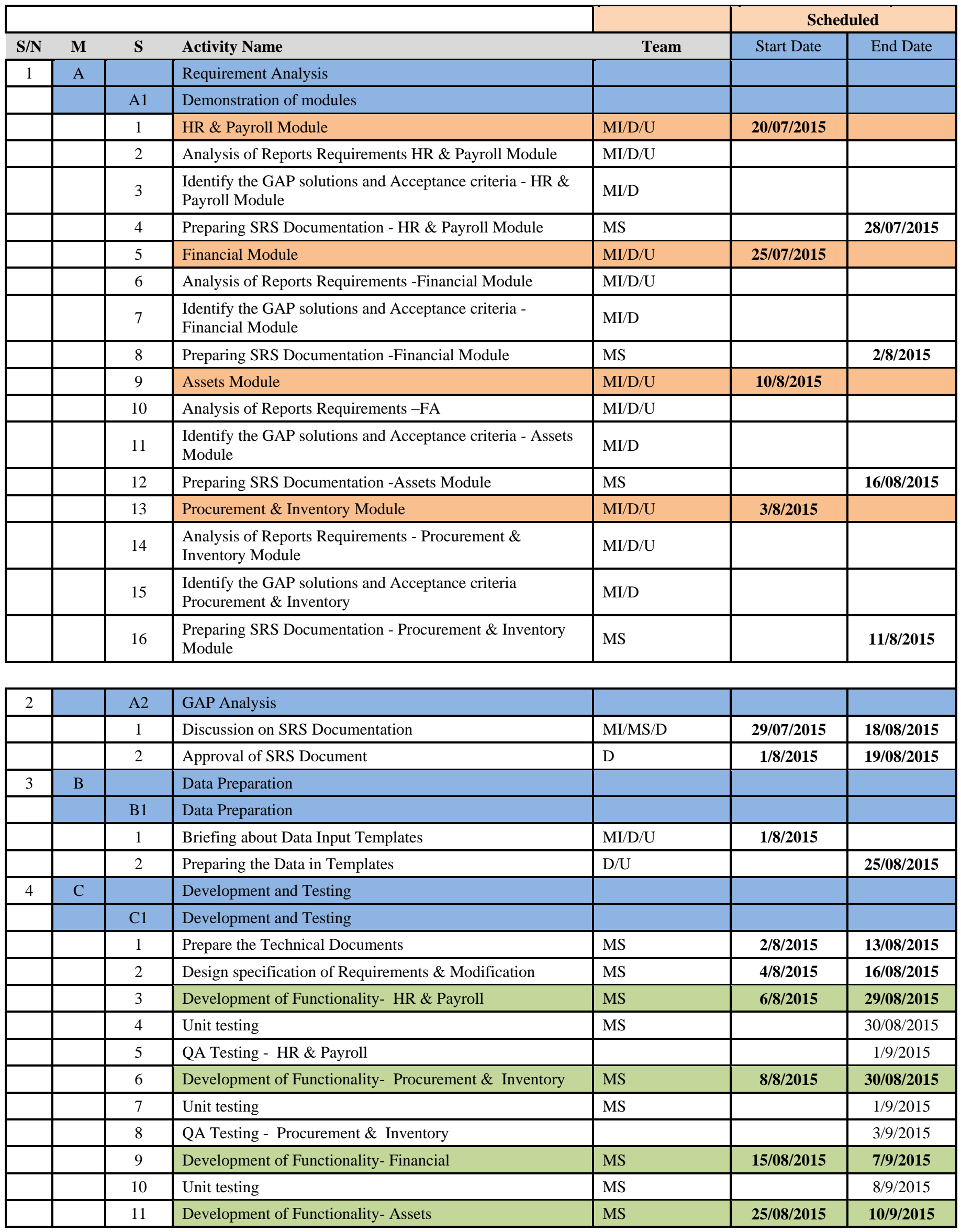




\begin{tabular}{|c|c|c|c|c|c|c|}
\hline & & 12 & Unit testing & MS & & $12 / 9 / 2015$ \\
\hline & & 13 & QA Testing - Financial \& Assets & MS/MI & & $15 / 09 / 2015$ \\
\hline & & 14 & System Testing [SIT]- HR \& Payroll & $\mathrm{MI} / \mathrm{D}$ & 2/9/2015 & 5/9/2015 \\
\hline & & 15 & System Testing [SIT]- Procurement \& Inventory & $\mathrm{MI} / \mathrm{D}$ & $6 / 9 / 2015$ & $8 / 9 / 2015$ \\
\hline & & 16 & System Testing [SIT] - Financial \& Assets & $\mathrm{MI} / \mathrm{D}$ & $19 / 09 / 2015$ & 22/09/2015 \\
\hline & & 17 & User Testing [UAT]- HR \& Payroll & $\mathrm{MI} / \mathrm{D} / \mathrm{U}$ & $14 / 09 / 2015$ & $19 / 09 / 2015$ \\
\hline & & 18 & User Testing [UAT]-Procurement \& Inventory & $\mathrm{MI} / \mathrm{D} / \mathrm{U}$ & $19 / 09 / 2015$ & $23 / 09 / 2015$ \\
\hline & & 19 & User Testing [UAT] - Financial \& Assets & $\mathrm{MI} / \mathrm{D} / \mathrm{U}$ & 26/09/2015 & $30 / 09 / 2015$ \\
\hline & & 20 & Feedback \& Final Release & MS/MI/D & $26 / 09 / 2015$ & $8 / 10 / 2015$ \\
\hline \multirow[t]{6}{*}{5} & $\overline{\mathrm{D}}$ & & Deployment Process & & & \\
\hline & & D1 & Database \& Application Deployment & & & \\
\hline & & 1 & Database Implementation & MI & $13 / 08 / 2015$ & \\
\hline & & 2 & Application Deployment -Procurement \& Inventory Module & MI & & 15/08/2015 \\
\hline & & 3 & Application Deployment-HR \& Payroll Module & MI & & $15 / 08 / 2015$ \\
\hline & & 4 & Application Deployment - -Financial \& Assets Module & MI & & $15 / 08 / 2015$ \\
\hline \multirow[t]{7}{*}{6} & $\overline{\mathrm{E}}$ & & Data Migration & & & \\
\hline & & E1 & Data Migration \& Verification & & & \\
\hline & & 1 & Verifying Data integrity & $\mathrm{MI} / \mathrm{D}$ & $20 / 08 / 2015$ & \\
\hline & & 2 & Uploading Inventory \&Procurement Master data & MI & $30 / 08 / 2015$ & $31 / 08 / 2015$ \\
\hline & & 3 & Uploading HR Master data & MI & $23 / 08 / 2015$ & $24 / 08 / 2015$ \\
\hline & & 4 & Uploading Accounts \& Asset Master data & MI & & $29 / 08 / 2015$ \\
\hline & & 5 & Integration of modules to Finance & $\mathrm{MI} / \mathrm{D} / \mathrm{U}$ & & $4 / 10 / 2015$ \\
\hline \multirow[t]{5}{*}{7} & $\mathrm{~F}$ & & Training, Finalizing \& Handover & & & \\
\hline & & F1 & Training & & & \\
\hline & & 1 & Training on Inventory \& Procurement Module & $\mathrm{MI} / \mathrm{D} / \mathrm{U}$ & $14 / 09 / 2015$ & $17 / 09 / 2015$ \\
\hline & & 2 & Training on HR\& Payroll Module & $\mathrm{MI} / \mathrm{D} / \mathrm{U}$ & 9/9/2015 & $13 / 09 / 2015$ \\
\hline & & 3 & Training on Accounts \& Assets Module & $\mathrm{MI} / \mathrm{D} / \mathrm{U}$ & 22/09/2015 & $27 / 09 / 2015$ \\
\hline \multirow[t]{14}{*}{8} & & $\mathrm{~F} 2$ & Finalizing \& Pilot Run & & & \\
\hline & & 1 & Finalizing Printing Formats - HR\& Payroll Module & $\mathrm{MI} / \mathrm{MS} / \mathrm{D} / \mathrm{U}$ & $10 / 10 / 2015$ & $11 / 10 / 2015$ \\
\hline & & 2 & $\begin{array}{l}\text { Finalizing Printing Formats - Inventory \&Procurement } \\
\text { Module }\end{array}$ & $\mathrm{MI} / \mathrm{MS} / \mathrm{D} / \mathrm{U}$ & $12 / 10 / 2015$ & 13/10/2015 \\
\hline & & 3 & Finalizing Printing Formats - Accounts \& Assets Module & $\mathrm{MI} / \mathrm{MS} / \mathrm{D} / \mathrm{U}$ & $14 / 10 / 2015$ & 15/10/2015 \\
\hline & & 4 & Finalizing User Access- HR\& Payroll Module & $\mathrm{MI} / \mathrm{D}$ & $17 / 10 / 2015$ & $18 / 10 / 2015$ \\
\hline & & 5 & Finalizing User Access - Inventory \&Procurement Module & $\mathrm{MI} / \mathrm{D}$ & $19 / 10 / 2015$ & 20/10/2015 \\
\hline & & 6 & Finalizing User Access - Accounts \& Assets Module & $\mathrm{MI} / \mathrm{D}$ & $21 / 10 / 2015$ & $22 / 10 / 2015$ \\
\hline & & 7 & Pilot Run- HR\& Payroll Module & $\mathrm{MI} / \mathrm{D} / \mathrm{U}$ & $24 / 10 / 2015$ & $5 / 11 / 2015$ \\
\hline & & 8 & Pilot Run- Inventory \&Procurement Module & $\mathrm{MI} / \mathrm{D} / \mathrm{U}$ & $24 / 10 / 2015$ & $5 / 11 / 2015$ \\
\hline & & 9 & Pilot Run- Accounts \& Assets Module & $\mathrm{MI} / \mathrm{D} / \mathrm{U}$ & $24 / 10 / 2015$ & $5 / 11 / 2015$ \\
\hline & & 10 & Review of Output & $\mathrm{D} / \mathrm{U}$ & $7 / 11 / 2015$ & $12 / 11 / 2015$ \\
\hline & & 11 & Live Run - HR\& Payroll Module & $\mathrm{D} / \mathrm{U}$ & & $14 / 11 / 2015$ \\
\hline & & 12 & Live Run - Other Module & $\mathrm{D} / \mathrm{U}$ & & $14 / 11 / 2015$ \\
\hline & & 13 & Sign Off & $\mathrm{MI} / \mathrm{D}$ & & $15 / 11 / 2015$ \\
\hline
\end{tabular}

D-Delivery Team, U- End USER, MI- Main IMPLEMENTATION Partner, MS- Main SOFTWARE DEVELOPMENT Team 
S/N

\begin{tabular}{|c|c|c|c|}
\hline 1 & A & & Requirement Analysis \\
\hline & & A1 & Demonstration of modules \\
\hline $\mathrm{P}$ & & 1 & HR \& Payroll Module \\
\hline$P$ & & & $\begin{array}{l}\text { Analysis of Reports Requirements -HR \& Payroll } \\
\text { Module }\end{array}$ \\
\hline 2 & & $\mathrm{~A} 2$ & GAP Analysis \\
\hline
\end{tabular}

\begin{tabular}{|c|c|c|l|}
\hline & & 1 & Identify the GAP solutions and Acceptance criteria \\
\hline & & 2 & Preparing SRS Documentation \\
\hline & & 3 & Discussion on SRS Documentation \\
\hline & & 4 & Approval of SRS Document \\
\hline 3 & B & & Data Preparation \\
\hline
\end{tabular}

\begin{tabular}{|c|c|c|l|l|}
\hline 3 & B & & Data Preparation & \\
\hline & & B1 & Data Preparation & \\
\hline & & 1 & Preparation of Data Int Templas \\
\hline
\end{tabular}

\begin{tabular}{|c|c|l|l|}
\hline $\mathrm{P}$ & & 1 & Preparation of Data Input Templates \\
\hline $\mathrm{P}$ & & 2 & Briefing about Data Input Templates \\
\hline $\mathrm{P}$ & & 3 & Preparing the Data in Templates \\
\hline
\end{tabular}

\begin{tabular}{|c|c|c|l|}
\hline $\mathrm{P}$ & & 3 & Preparing the Data in Templates \\
\hline $\mathrm{P}$ & & 4 & Data Template Verification \\
\hline 4 & $\mathrm{C}$ & & Development and Testing \\
\hline & & $\mathrm{C} 1$ & Development and Testing \\
\hline $\mathrm{P}$ & & 1 & Development of Functionality- \\
\hline
\end{tabular}

\begin{tabular}{|c|c|c|}
\hline & $\mathrm{C} 1$ & Development and Testing \\
\hline $\mathrm{P}$ & 1 & Development of Functionality- \\
\hline $\mathrm{P}$ & 2 & Unit testing \\
\hline $\mathrm{P}$ & 3 & Development of Functionality Country Specific \\
\hline $\mathrm{P}$ & 4 & Unit testing \\
\hline
\end{tabular}

\begin{tabular}{|l|l|l|l|l|}
\hline $\mathrm{P}$ & & 4 & Unit testing & $\mathrm{MS}$ \\
\hline $\mathrm{P}$ & & 5 & QA Testing & MS/MI \\
\hline $\mathrm{P}$ & & 6 & User Testing [UAT] & $\mathrm{MI} / \mathrm{D} / \mathrm{U}$ \\
\hline $\mathrm{P}$ & & 7 & Feedback \& Final Release & $\mathrm{MS} / \mathrm{MI} / \mathrm{D}$ \\
\hline 5 & $\mathrm{D}$ & & Deployment Process & \\
\hline & & $\mathrm{D}$ & Dabas \& Application Deployt & \\
\hline
\end{tabular}

.

\begin{tabular}{|c|c|c|c|c|c|c|c|c|c|c|}
\hline 6 & $\mathrm{E}$ & & Data Migration & & & & & & & \\
\hline & & E1 & Data Migration \& Verification & & 15 & 7 & 7 & 7 & 7 & 7 \\
\hline & & 1 & Verifying Data integrity & $\mathrm{MI} / \mathrm{D}$ & & & & & & \\
\hline & & 2 & Uploading Inventory \&Procurement Master data & MI & & & & & & \\
\hline & & 3 & Uploading HR Master data & MI & & & & & & \\
\hline & & 4 & Uploading Accounts \& Asset Master data & MI & & & & & & \\
\hline & & 5 & Integration of modules to Finance & $\mathrm{MI} / \mathrm{D} / \mathrm{U}$ & & & & & & \\
\hline 7 & $\mathrm{~F}$ & & Training, Finalizing \& Handover & & & & & & & \\
\hline
\end{tabular}




\begin{tabular}{|c|c|c|c|c|c|c|c|c|c|}
\hline & $\mathrm{F} 1$ & Training & \multicolumn{2}{|r|}{10} & 10 & 10 & 10 & 10 & 10 \\
\hline & 1 & Training on Procurement Module & $\mathrm{MI} / \mathrm{D} / \mathrm{U}$ & & & & & & \\
\hline & 2 & Training on Inventory Module & $\mathrm{MI} / \mathrm{D} / \mathrm{U}$ & & & & & & \\
\hline & 3 & Training on Accounts \& Assets Module & $\mathrm{MI} / \mathrm{D} / \mathrm{U}$ & & & & & & \\
\hline & 4 & Training on HR\& Payroll Module & $\mathrm{MI} / \mathrm{D} / \mathrm{U}$ & & & & & & \\
\hline 8 & $\mathrm{~F} 2$ & Finalizing \& Pilot Run & & 60 & 14 & 14 & 14 & 14 & 14 \\
\hline & 1 & Finalizing Printing Formats & $\mathrm{MI} / \mathrm{MS} / \mathrm{D} / \mathrm{U}$ & & & & & & \\
\hline & 2 & Finalizing User Access & $\mathrm{MI} / \mathrm{D}$ & & & & & & \\
\hline & 3 & Pilot Run & $\mathrm{MI} / \mathrm{D} / \mathrm{U}$ & & & & & & \\
\hline & 4 & Review of Output & $\mathrm{MI} / \mathrm{D} / \mathrm{U}$ & & & & & & \\
\hline & 5 & Live Run & $\mathrm{D} / \mathrm{U}$ & & & & & & \\
\hline & 6 & Sign Off & $\mathrm{MI} / \mathrm{D}$ & & & & & & \\
\hline & & Total Days & & 260 & 111 & 111 & 111 & 111 & 111 \\
\hline
\end{tabular}

D-Delivery Team, U- End USER, MI- Main IMPLEMENTATION Partner, MS- Main SOFTWARE DEVELOPMENT Team

P Parallel Run 